\title{
BMJ Open Can clinical features be used to differentiate type 1 from type 2 diabetes? A systematic review of the literature
}

\author{
Beverley M Shields, ${ }^{1}$ Jaime L Peters, ${ }^{2}$ Chris Cooper, ${ }^{2}$ Jenny Lowe, ${ }^{2}$ \\ Bridget A Knight, ${ }^{1,3}$ Roy J Powell, ${ }^{3}$ Angus Jones, ${ }^{1}$ Christopher J Hyde, ${ }^{2}$ \\ Andrew T Hattersley ${ }^{1}$
}

To cite: Shields BM, Peters JL, Cooper C, et al. Can clinical features be used to differentiate type 1 from type 2 diabetes? A systematic review of the literature. $B M J$ Open 2015;5:e009088. doi:10.1136/bmjopen-2015009088

- Prepublication history and additional material is available. To view please visit the journal (http://dx.doi.org/ 10.1136/bmjopen-2015009088).

Received 15 June 2015 Revised 10 August 2015 Accepted 2 September 2015

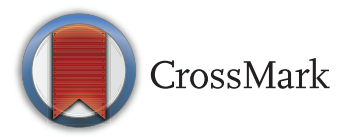

${ }^{1}$ Department of NIHR Exeter Clinical Research Facility, University of Exeter Medical School, University of Exeter, Exeter, UK

${ }^{2}$ Department of Evidence Synthesis \& Modelling for Health Improvement (ESMI) University of Exeter Medical School, University of Exeter, Exeter, UK

${ }^{3}$ Department of Research and Development, Royal Devon and Exeter NHS Foundation Trust, Exeter, UK

Correspondence to Professor Andrew T Hattersley;

A.T.Hattersley@exeter.ac.uk

\section{ABSTRACT}

Objective: Clinicians predominantly use clinical features to differentiate type 1 from type 2 diabetes yet there are no evidence-based clinical criteria to aid classification of patients. Misclassification of diabetes is widespread (7-15\% of cases), resulting in patients receiving inappropriate treatment. We sought to identify which clinical criteria could be used to discriminate type 1 and type 2 diabetes.

Design: Systematic review of all diagnostic accuracy studies published since 1979 using clinical criteria to predict insulin deficiency (measured by C-peptide).

Data sources: 14 databases including: MEDLINE, MEDLINE in Process and EMBASE. The search strategy took the form of: (terms for diabetes) AND (terms for C-Peptide).

Eligibility criteria: Diagnostic accuracy studies of any routinely available clinical predictors against a reference standard of insulin deficiency defined by cutoffs of C-peptide concentrations. No restrictions on race, age, language or country of origin.

Results: 10917 abstracts were screened, and 231 full texts reviewed. 11 studies met inclusion criteria, but varied by age, race, year and proportion of participants who were C-peptide negative. Age at diagnosis was the most discriminatory feature in $7 / 9$ studies where it was assessed, with optimal cut-offs ( $>70 \%$ mean sensitivity and specificity) across studies being $<30$ years or $<40$ years. Use of/time to insulin treatment and body mass index (BMI) were also discriminatory. When combining features, BMI added little over age at diagnosis and/or time to insulin $(<1 \%$ improvement in classification).

Conclusions: Despite finding only 11 studies, and considerable heterogeneity between studies, age at diagnosis and time to insulin were consistently the most discriminatory criteria. BMI, despite being widely used in clinical practice, adds little to these two criteria. The criteria identified are similar to the Royal College of General Practitioners National Health Service (RCGP/NHS) Diabetes classification guidelines, which use age at diagnosis $<35$ years and time to insulin $<6 \mathrm{~m}$. Until further studies are carried out, these guidelines represent a suitable classification scheme.

\section{Strengths and limitations of this study}

- We have carried out a comprehensive and robust systematic review in accordance with PRISMA guidelines and our initial published protocol.

- We screened a large number of literature sources, and all reviewing and data extraction was carried out in duplicate independently by two authors (BS and JP).

- Considerable heterogeneity across studies precluded a formal meta-analysis.

- A limited number of studies were found meaning there is still considerable uncertainty around criteria for classification of type 1 and type 2 diabetes.

- Variability in the reference standard of insulin deficiency across studies also led to further uncertainty around findings limiting direct usefulness of criteria.

Systematic review registration: PROSPERO reference CRD42012001736.

\section{BACKGROUND}

Correct classification of a patient's diabetes is crucial for ensuring they receive the most appropriate treatment and management. Current guidelines for the treatment of diabetes are specific to type 1 and type 2 diabetes (T1D and T2D) and these show marked differences, ${ }^{1-4}$ reflecting the difference in endogenous insulin production between the two subtypes. Patients with T1D rapidly develop severe insulin deficiency, leading to high glycemic instability and so require accurate insulin replacement (such as multiple injections and carbohydrate counting), and have poor response to noninsulin therapies. ${ }^{3} 5$ Patients with T2D still 
continue to produce substantial amounts of their own insulin, and, therefore, respond to non-insulin therapy, have more stable glycaemia and, if insulin treatment is needed, may achieve good control with nonphysiological insulin regimes. ${ }^{6} 7$

Currently, there are no published, evidence-based, guidelines or criteria for diabetes classification, despite the importance for patient management. Guidance on the classification of the two types of diabetes from major health organisations is limited, and focuses on aetiology, ${ }^{8} 9$ whereas it is insulin production that is the driver for informing treatment decisions. Insulin deficiency/production can be assessed by measurement of C-peptide in either blood or urine, ${ }^{10}$ but it is rarely measured in clinical practice and current guidelines for diabetes management do not recommend its routine use. ${ }^{1}{ }^{111}$ Classification is based primarily on clinical judgement, with younger slimmer patients tending to be classed as T1, and older, more obese patients diagnosed as $\mathrm{T} 2 .{ }^{8}$ However, with obesity increasing in the population and the resulting increase in T2D in the young, this traditional distinction has become less clear. ${ }^{12} 13$

Misclassification of diabetes has been shown to occur in $7-15 \%$ of cases, ${ }^{13-15}$ and these studies are likely to underestimate the problem, as they only use clinical 'clues' as their reference standard. The current practice based on aetiological guidelines and clinical opinion is clearly insufficient. Pragmatic guidelines on diabetes classification have been developed by National Health Service (NHS) Diabetes and The Royal College of General Practitioners (RCGP) in the UK, but are taken from consensus expert clinical opinion rather than being evidence-based. ${ }^{13}$

In order to determine evidence-based criteria that could be used to classify the two main forms of diabetes, an appropriate gold standard is necessary. The most important reason for correctly classifying patients is to ensure appropriate treatment and management, and the main factor determining this is the difference in endogenous insulin production between patients with $\mathrm{T} 1$ and T2D. Therefore, long-term insulin deficiency represents an acceptable reference standard for T1D. This is likely to be preferable to using markers of the autoimmune process associated with T1D. While measurement of various islet autoantibodies may aid discrimination, these are imperfect measures, ${ }^{16}$ and most importantly, the presence of islet autoimmunity does not in itself determine treatment requirement. ${ }^{17}$

We aimed to systematically review the literature to identify clinical criteria, predictive of severe insulin deficiency, that could be used to discriminate T1D and T2D and inform evidence-based guidelines for the classification of diabetes.

\section{METHODS}

We followed the PRISMA guidelines for the reporting of systematic reviews. The original protocol has been published ${ }^{18}$ and is registered on PROSPERO (http://www.crd.york.ac. uk/PROSPERO/reference CRD4201200173 6).

\section{Data sources and search strategy}

Fourteen databases were searched systematically: MEDLINE, MEDLINE in Process, EMBASE, PsycINFO, Social Policy and Practice, AMED, British Nursing Index, CINAHL, HMIC, Sociological Abstracts, ASSIA, Cochrane, Web of Science, Centre for Reviews and Dissemination). The search strategy took the form of: (terms for diabetes) AND (terms for C-Peptide). Searches were limited to human only populations and from 1979 since that was when the original classification scheme was proposed by the National Diabetes Data Group. ${ }^{19}$ Searches were not limited by language or study design.

Searches were also carried out on the Conference Proceedings Citation Index as well as the proceedings of the American Diabetes Association, the European Association for the Study of Diabetes, and Diabetes UK. BL Ethos was also searched for theses. Web-searching was conducted, including web-site specific searches of WHO and NICE. Forwards and backwards citation chasing was conducted on all studies included at fulltext. The full search strategies are recorded in the online supplementary Search Annex. Searches were initially performed in October 2012 and were updated on 3 April 2014 to capture any additional studies that may have been carried out since the beginning of the review.

\section{Study selection}

A two-stage screening process was undertaken. In Stage 1 , after removing duplicates, two reviewers (BMS and JLP) independently screened the titles and abstracts of all references against the inclusion and exclusion criteria. In Stage 2, full texts were retrieved on all studies included at the first screening stage and were independently screened (by BMS and JLP). Authors of included conference abstracts were searched to determine whether a full article had subsequently been published. Any discrepancies between the two reviewers were discussed and resolved by consensus, or in discussion with a third reviewer (RJP).

\section{Inclusion and exclusion criteria}

Included studies comprised diagnostic accuracy studies of clinical predictors of insulin deficiency, with the reference standard of insulin deficiency being defined by cut-offs of C-peptide results. All measurements of C-peptide and all cut-offs for insulin deficiency were included. Clinical predictors were defined as any routinely measured clinical feature and studies were eligible if there was a cut-off for that clinical predictor assessed against the measure of insulin deficiency. There were no restrictions on race, age or country of origin. Studies examining islet autoantibodies only were excluded as they are not routinely measured. A separate systematic review examining the diagnostic accuracy of islet 
autoantibodies is presently underway (Prospero reference CRD42012001736). Studies where patients had known causes of diabetes, for example, monogenic, secondary or syndromic diabetes, were excluded.

\section{Data extraction}

For all studies meeting the inclusion and exclusion criteria, data were extracted independently by both reviewers (BMS and JLP). Data extraction forms were developed and piloted prior to the review. Key details of population (age, sex, country, race, year), diabetes (definition of diabetes, treatment, subgroups), reference standard (type of sample, stimulation, assay, cut-off used) and clinical predictors (which predictors were included, how they were measured, the cut-offs used) were recorded. All C-peptide cut-offs were converted to the fasting serum equivalent to allow direct comparison. ${ }^{10}$ Two-by-two tables were extracted where possible to determine the proportion of patients who were C-peptide negative/positive (ie, below/above the cut-off) and the sensitivity, specificity, positive and negative predictive values of the clinical characteristics at reported cut-offs.

\section{Quality assessment}

Both reviewers (BMS and JLP) assessed quality independently and discrepancies were resolved by consensus. Quality assessment forms, based on the criteria set out in QUADAS-2, ${ }^{20}$ were developed and piloted prior to review. These criteria included assessment of internal and external validity of patient selection, the clinical predictors and patient flow and timing. Variability in the measurements for the reference standard was assessed separately. Further details are available in the online supplementary material.

\section{Data synthesis}

Owing to the considerable heterogeneity between the studies identified, meta-analysis, as proposed in our original protocol, was not appropriate. Data synthesis is, therefore, largely descriptive with summary data presented. Criteria with a mean of sensitivity and specificity $>70 \%$ (equivalent to a receiver operating characteristic area under curve of 0.7 ) were considered clinically useful. Ranking of the discriminatory ability of criteria within studies was used to compare their relative performance.

\section{Reporting bias}

No formal assessment of publication bias was undertaken due to heterogeneity between studies and the small number of included studies. We did perform a comprehensive and exhaustive search including grey literature, however it cannot be ruled out that our systematic review is affected by reporting biases.
RESULTS

\section{Initial screening}

Figure 1 shows the flow diagram of citations found. A total of 10917 records were identified from database searches and a further 148 sources were identified from grey literature searches. After title and abstract screening, 194 articles were deemed potentially relevant. Following full-text screening, nine studies were identified as eligible based on our inclusion criteria ${ }^{21-29}$ (for further details see online supplementary material).

Backward and forward citation searching was carried out on the nine included references, and conference abstracts were followed up, identifying a further 43 studies for fulltext review, one of which ${ }^{30}$ met our inclusion criteria. In April 2014, an update search was performed yielding a further 2101 references for screening. Thirty-six of these were identified by the two reviewers as requiring full-text review, and one of these fitted inclusion criteria. ${ }^{31}$ Thus, 11 articles contribute to this systematic review.

\section{Data extraction and quality assessment}

There was considerable heterogeneity across the included studies (see online supplementary table S1). The 11 included studies spanned a wide range of years (19812013). Studies varied in terms of race, age group and subgroups of diabetes studied. One study included only patients with end-stage renal disease, ${ }^{22}$ whereas it was a specific exclusion criterion for another study. ${ }^{28}$ Three studies focused on insulin-treated patients only, ${ }^{24} 2930$ whereas the other studies either included all patients regardless of treatment or did not report on treatment. Sample size ranged from $<100^{22} 2931$ to $>3000 .^{25}$ The proportion of patients classified as insulin deficient (based on the reported C-peptide cut-off in each paper) also varied (median (range) 40\% (7-69\%)), reflecting differing inclusion criteria across studies altering the proportions with different forms of diabetes across the studies.

Quality assessment of the included studies is summarised in online supplementary table S2. In general, there was a low risk of bias in terms of patient selection and patient flow/timing. Two studies were at high risk of bias $^{22} 29$ in terms of the clinical criteria used as these were internally derived, so diagnostic performance is likely overestimated in these cases. ${ }^{32}$ In terms of external validity, studies were all applicable to our broad research question but most restricted inclusion criteria to a subset of the diabetic population.

The reference standards varied in terms of sample provided, timing of sample in relation to meal stimulation, and cut-offs for C-peptide (see online supplementary table S3). Five studies report deriving their cut-offs from previous papers. ${ }^{21} 22252930$ Two studies derived the cut-off used from their own data, ${ }^{27}{ }^{28}$ potentially introducing bias, although the cut-offs were comparable to those derived from the literature. Despite the variation in measurements, all were appropriate to classify insulin deficiency and cut-offs were largely comparable with most approximating $0.2 \mathrm{nmol} / \mathrm{L},,^{21} 2224-262830$ and 


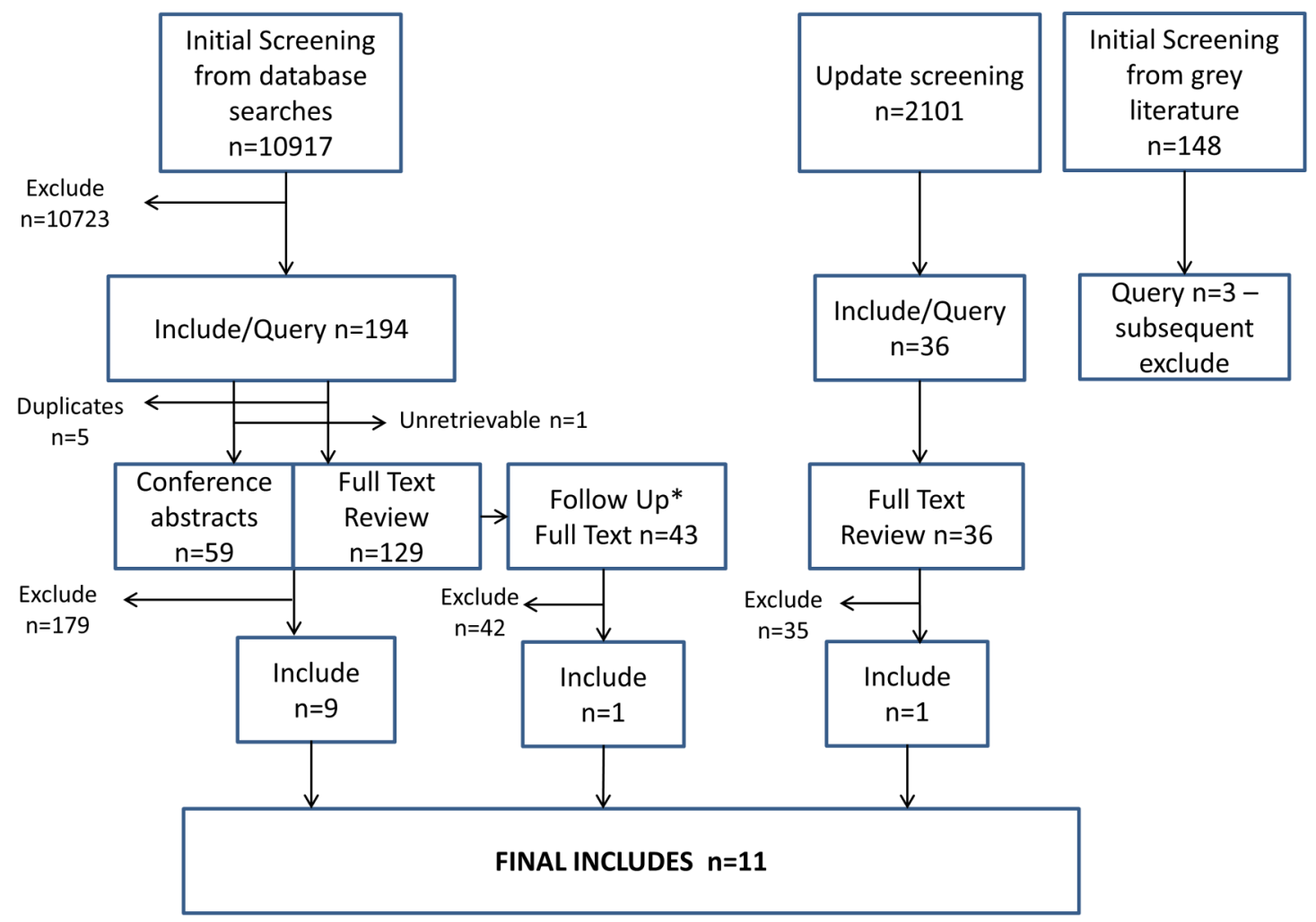

Figure 1 Flow diagram showing inclusions and exclusions from title and abstract screening, and full-text review. *Follow-Up includes full texts identified from follow-up of conference abstracts $(n=29)$ and references identified from backwards and forwards citation chasing $(n=14)$.

four studies using a slightly more conservative cut-off $(0.03-0.08 \mathrm{nmol} / \mathrm{L}){ }^{23} 272931$ Only one study measured C-peptide and clinical features at diabetes diagnosis. ${ }^{31}$ All other studies were cross-sectional with varying duration of diabetes.

\section{Data synthesis}

Owing to the heterogeneity across studies, particularly in terms of inclusion criteria, formal quantitative meta-analysis was not appropriate. Therefore, data synthesis is largely descriptive.

Age at diagnosis, BMI, insulin treatment/time to insulin are consistent predictors of insulin deficiency across studies

Age at diagnosis (9 studies), measures of obesity (including BMI, or percentage desirable weight in earlier studies) (8 studies) and either time to insulin treatment

Table 1 Criteria reported in the 11 included studies used to discriminate between C-peptide positive and negative patients

\begin{tabular}{|c|c|c|c|c|c|}
\hline First author (year) & $\begin{array}{l}\text { Age at } \\
\text { diagnosis }\end{array}$ & $\begin{array}{l}\text { Insulin treated or } \\
\text { Time to insulin }\end{array}$ & $\begin{array}{l}\text { BMI } \\
\text { (or similar) }\end{array}$ & DKA & $\begin{array}{l}\text { Onset (gradual } \\
\text { or acute) }\end{array}$ \\
\hline Prior (1991) & 1 & 2 & 3 & & \\
\hline Welborn (1983) & 2 & 1 & 3 & & \\
\hline Laakso (1987) & 3 & 1 & 2 & & \\
\hline Benhamou (1992) & 1 & 2 & 3 & $x$ & $x$ \\
\hline Shields (2010) & 1 & 2 & 3 & & \\
\hline Service (1997) & \# & \# & \# & \# & \# \\
\hline Boyle (1999) & 1 & 2 & 3 & & \\
\hline Welborn (1981) & 1 & 2 & & & \\
\hline Nielsen (1986) & 1 & & & & \\
\hline Ekpebegh (2013) & 1 & & 2 & Inc & \\
\hline Balasubramanyam (2006) & & & 1 & Inc & \\
\hline
\end{tabular}


(5 studies) and/or use of insulin treatment (3 studies) were identified as consistent clinical criteria predictive of insulin deficiency (table 1). In all studies reporting these criteria, younger age at diagnosis, slimmer BMI and shorter time to insulin was used to define insulin deficiency.

Absence of each of acanthosis nigricans and hypertension were predictive of insulin deficiency (overall correct classification rates of $61 \%$ and $72 \%$, respectively), but these were only assessed in one study. ${ }^{31}$ Other measures were available in four studies ${ }^{22} 262731$ (including history of diabetic ketoacidosis (DKA) ${ }^{22} 26$ or ketonuria, ${ }^{22}$ history of hypoglycaemia, ${ }^{27}$ speed of onset of diabetes, ${ }^{26}$ long-term complications, ${ }^{22}$ polyuria, ${ }^{22}$ weight loss, ${ }^{22}$ post-Sustacal glucose, ${ }^{27}$ serum creatinine, ${ }^{27}$ diabetes in a first-degree relative ${ }^{31}$ and history of poor $\operatorname{control}^{27}$ ), but they were either not discriminatory, or they contributed very little individual discriminatory power to an overall algorithm.

\section{Age at diagnosis cut-offs better predicted insulin} deficiency than cut-offs of BMI or time to insulin

When comparing discriminative ability of the most commonly reported criteria within studies, age at diagnosis, at the cut-off described in the individual study, correctly classified more patients than the other clinical features (most discriminatory criteria in $7 / 9$ studies). Time to insulin/insulin treatment was the next best predictor, and BMI (or equivalent) was the weakest of the significant predictors (table 1).

\section{Cut-offs for age at diagnosis, BMI and time to insulin were fairly consistent across studies}

Cut-offs with the best combination of sensitivity and specificity (mean of sensitivity and specificity $>70 \%$ ) were similar across studies. For predicting insulin deficiency, the best cut-offs for age at diagnosis were $<30$ years $(2$ studies) or $</ \leq 40$ years (4 studies). For time to insulin, $<1$ year (1 study) or $</ \leq 2$ years (2 studies) were the best cut-offs, although longer cut-offs were not assessed in any of the studies identified. For BMI, cut-offs $<27 \mathrm{~kg} / \mathrm{m}^{2}$ (1 study) and $<28 \mathrm{~kg} / \mathrm{m}^{2}$ (3 studies) were most useful (see table 2 ). Extracted $2 \times 2$ tables are presented online supplementary tables 4 .

\section{BMI cut-offs provide little improvement in classification in addition to age at diagnosis and insulin use/time to insulin criteria}

Combinations of cut-offs did not consistently improve the overall rate of classification. The addition of BMI did not improve classification over age at diagnosis and/or use of/time to insulin treatment in all five studies where these combinations were reported $(<1 \%$ improvement in classification; see table 3). The addition of insulin treatment or time to insulin criteria improved classification over using age at diagnosis alone in $3 / 5$ studies where both were reported (see table 3 ). Extracted $2 \times 2$ tables and summary statistics are presented in online supplementary tables S4 and S5.

\section{DISCUSSION}

\section{Principal findings}

Few studies have robustly assessed utility of clinical features in diagnosing diabetes subtype

There were only 11 appropriate studies that examined which clinical characteristics could discriminate between T1 and T2D, using the reference standard of insulin deficiency. This is a remarkably low number of studies considering the vast majority of the $>200$ million patients with diabetes will be classified into type 1 or type 2 on the basis of clinical features alone and an incorrect classification will result in inappropriate treatment.

\section{Age at diagnosis was the most discriminatory clinical feature}

Age at diagnosis, time to insulin and BMI consistently emerged as the main discriminatory clinical criteria despite the considerable heterogeneity of the included studies. Age at diagnosis was the best discriminatory criteria with diagnosis either below 30 or below 40 years being predictive of T1D. In terms of providing useful criteria for clinical practice, based on the current available evidence, this would suggest clinicians should place more emphasis on age than obesity when diagnosing diabetes subtype, but exercise caution when classifying patients diagnosed between the ages of 30 and 40 where further investigation is likely to be necessary.

\section{Time to insulin treatment is a useful discriminator, but} biased by physician opinion

Starting insulin treatment before 2 years did slightly improve discrimination over age of diagnosis (table 3 ). However, treatment assignment can clearly not be used to define initial treatment, which is one of the major reasons for determining diabetes subtype. Treatment decisions are physician-dependent, as well as diseasedependent, so will vary between clinicians.

\section{BMI discriminatory but adds little over age at diagnosis}

BMI provided $<1 \%$ improvement in classification over age at diagnosis or age at diagnosis and time to insulin. Clinicians often use obesity as a marker to indicate T2D, but our findings suggest using this is unlikely to be helpful over and above using age at diagnosis.

\section{Other may not be sufficiently discriminatory}

Other measures were less often studied. Acanthosis nigricans and hypertension did discriminate C-peptide positive from C-peptide negative patients, but these were only assessed in one study. Other features were either not discriminatory or only contributed weakly to an algorithm, and therefore unlikely to be useful in practice. These measures included features of diagnosis such as diabetic ketoacidosis, ketonuria and rapid onset of symptoms including weight loss. In fact, in the two studies 
Table 2 Criteria for predicting type 1 diabetes-single criteria

\begin{tabular}{|c|c|c|c|c|c|c|c|c|c|}
\hline Cut-off & Author (year) & $\mathbf{N}$ & $\begin{array}{l}\text { Per cent } \\
\text { C-pep } \\
\text { neg }\end{array}$ & $\begin{array}{l}\text { Sens } \\
(\%)\end{array}$ & $\begin{array}{l}\text { Spec } \\
(\%)\end{array}$ & $\begin{array}{l}\text { Mean sens } \\
\text { and spec }\end{array}$ & $\begin{array}{l}\text { Per cent } \\
\text { correct }\end{array}$ & PPV & NPV \\
\hline \multicolumn{10}{|c|}{ (i) Age at diagnosis (a/d) } \\
\hline$<20$ & Boyle (1999) & 3613 & 7 & 20 & 97 & 59 & 92 & 36 & 94 \\
\hline$\leq \mathbf{3 0}$ & Prior (1991) & 575 & 61 & 84 & 82 & 83 & 83 & 88 & 77 \\
\hline$<30$ & Nielsen (1986) & 215 & 69 & 64 & 88 & 76 & 72 & 92 & 53 \\
\hline$<30$ & Ekpebegh (2013) & 71 & 49 & 57 & 72 & 65 & 65 & 67 & 63 \\
\hline$<39$ & Shields (2010) & 72 & 56 & 68 & 97 & 83 & 81 & 96 & 70 \\
\hline$\leq 40$ & Prior (1991) & 575 & 61 & 97 & 59 & 78 & 82 & 79 & 92 \\
\hline$\leq 40$ & Welborn (1983) & 121 & 21 & 84 & 85 & 85 & 85 & 60 & 95 \\
\hline$\leq 40$ & Welborn (1981) & 201 & 24 & 76 & 81 & 79 & 79 & 55 & 92 \\
\hline$\leq 40$ & Laakso* (1987) & 171 & 67 & 61 & 79 & 70 & 67 & 85 & 44 \\
\hline$<45$ & Boyle (1999) & 3613 & 7 & 65 & 57 & 61 & 57 & 10 & 96 \\
\hline \multicolumn{10}{|c|}{ (ii) Insulin treatment/time to insulin (tti) ( $a=a$ ll treatments, $i=$ insulin-treated only) } \\
\hline $\begin{array}{l}\text { on insulin } \\
\text { (a) }\end{array}$ & Prior (1991) & 575 & 61 & 99 & 25 & 62 & 70 & 68 & 97 \\
\hline $\begin{array}{l}\text { on insulin } \\
\text { (a) }\end{array}$ & Welborn (1981) & 201 & 24 & 100 & 70 & 85 & 77 & 49 & 100 \\
\hline $\begin{array}{l}\text { on insulin } \\
\text { (a) }\end{array}$ & Boyle (1999) & 3613 & 7 & 91 & 61 & 76 & 63 & 15 & 99 \\
\hline $\mathrm{tti} \leq 1.5 \mathrm{~m}(\mathrm{i})$ & Shields (2010) & 72 & 56 & 80 & 56 & 68 & 69 & 70 & 69 \\
\hline tti<1y (a) & Prior (1991) & 575 & 61 & 92 & 75 & 84 & 85 & 85 & 85 \\
\hline$t t i<2 y(a)$ & Welborn (1983) & 121 & 21 & 100 & 82 & 91 & 86 & 60 & 100 \\
\hline $\begin{array}{l}\mathrm{tti} \leq 2 \mathrm{y} \text { (i) } \\
\text { (iii) } \mathrm{BMI}\end{array}$ & Laakso* (1987) & 90 & 67 & 70 & 86 & 78 & 75 & 91 & 58 \\
\hline$<20$ & Boyle (1999) & 3613 & 7 & 10 & 98 & 54 & 92 & 33 & 94 \\
\hline$<25 \dagger$ & Prior (1991) & 575 & 61 & 34 & 92 & 63 & 57 & 87 & 47 \\
\hline$<25$ & Boyle (1999) & 3613 & 7 & 41 & 86 & 64 & 83 & 18 & 95 \\
\hline$<27 \dagger$ & Prior (1991) & 575 & 61 & 87 & 63 & 75 & 78 & 79 & 76 \\
\hline$\leq \mathbf{2 7} \dagger$ & Welborn (1983) & 121 & 21 & 80 & 67 & 74 & 69 & 38 & 93 \\
\hline$\leq 27$ & Laakso* (1987) & 90 & 67 & 76 & 66 & 71 & 73 & 82 & 57 \\
\hline$<28$ & $\begin{array}{l}\text { Balasumbryaman } \\
(2006)\end{array}$ & 294 & 60 & 67 & 86 & 77 & 78 & 79 & 77 \\
\hline$<29$ & Boyle (1999) & 3613 & 7 & 71 & 57 & 64 & 58 & 11 & 96 \\
\hline$<29$ & Shields (2010) & 72 & 56 & 78 & 56 & 67 & 68 & 69 & 67 \\
\hline$<30$ & Ekpebegh (2013) & 71 & 49 & 77 & 47 & 62 & 62 & 59 & 68 \\
\hline \multicolumn{10}{|c|}{$\begin{array}{l}\text { Sensitivity (sens), specificity (spec), proportion correctly classified (\%correct), mean of sensitivity and specificity (mean sens and spec), } \\
\text { positive predictive value (PPV), and negative predictive value (NPV) for (i) age at diagnosis, (ii) body mass index (BMI) and (iii) insulin } \\
\text { treatment and/or time to insulin. Proportion of C-peptide negative patients (\% C-pep neg) shown to aid interpretation of \% correct, PPV and } \\
\text { NPV. Criteria with a mean sensitivity and specificity }>70 \% \text { are highlighted in bold. } \\
{ }^{*} \text { Male and female values combined, using postglucagon-stimulated results. } \\
\text { †Converted from percentage desirable weight. }\end{array}$} \\
\hline
\end{tabular}

examining only patients presenting with DKA, $40 \%$ and $46 \%$ were C-peptide positive, ${ }^{21}{ }^{31}$ suggesting DKA is not useful in its own right for classifying a patient as having type 1 diabetes.

\section{Strengths and weaknesses \\ Strengths}

We have carried out a comprehensive and robust systematic review in accordance with PRISMA guidelines and our initial published protocol. ${ }^{18}$ We screened a large number of literature sources, and all reviewing and data extraction was carried out in duplicate independently by two authors (BMS and JLP).

\section{Limitations}

Heterogeneity across studies could have influenced the diagnostic performance of cut-offs identified and so precluded formal meta-analysis. There were four key areas in particular, where heterogeneity was apparent: (1) The proportion of insulin-deficient patients varied considerably across the studies (range 7-69\%), reflecting major differences in inclusion criteria for each study and varying proportions of $\mathrm{T} 1$ and $\mathrm{T} 2 \mathrm{D}$ in the study populations. (2) Studies spanned over 30 years (1981-2013) and there have been considerable changes in the phenotype of T1 and T2D in this time. With the rising prevalence of obesity in the population, T1 patients are now more likely to be obese than in the past, and T2D has 


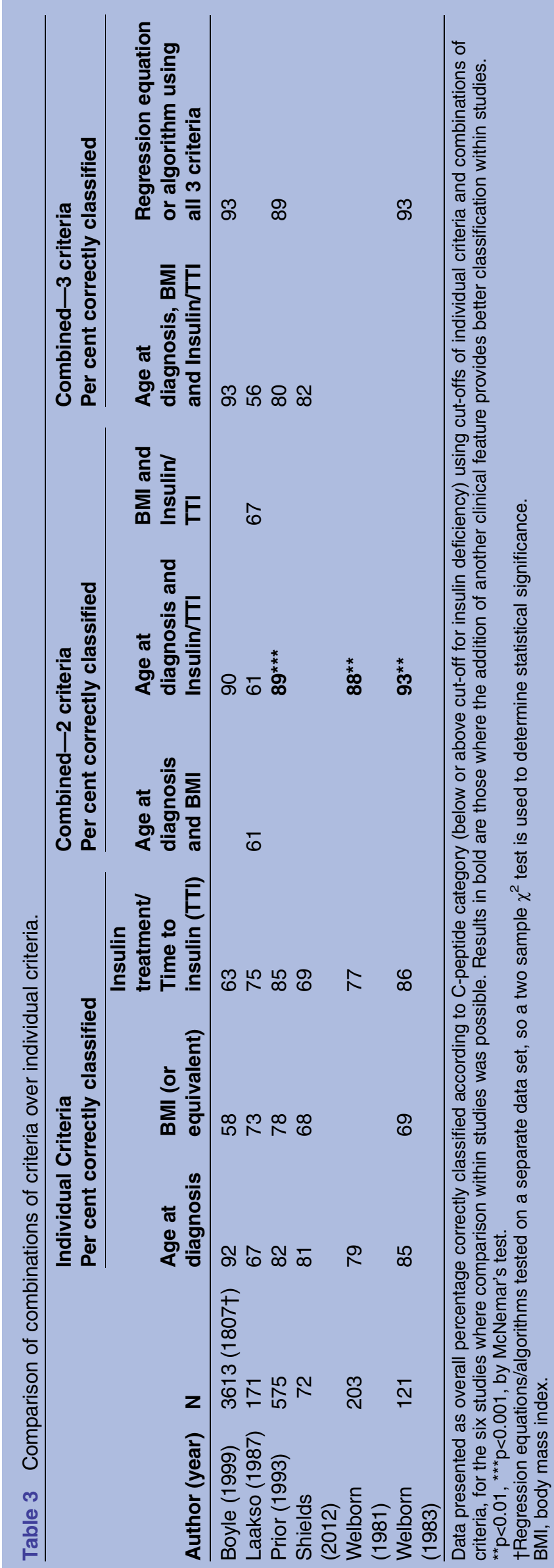

become more common in young adults. (3) Renal disease is known to impact on C-peptide clearance, so differences were likely in the studies excluding patients with renal disease, ${ }^{25} 28$ compared with those exclusively examining those with ESRD. ${ }^{22}$ (4) Ethnicity differed across studies, from populations that were predominantly Caucasian, ${ }^{23} 27$ to those predominantly Hispanic and/or Black African ${ }^{31}$ /African-American patients. ${ }^{21} 25$ Despite the considerable differences in studies, however, there were consistencies in the criteria identified and the most discriminatory cut-offs across the different populations.

The small number of studies and the heterogeneity between them means there is still uncertainty around the usefulness of the criteria and cut-offs proposed, and highlights a clear need for further work in this area. This review provides a strong starting point from which to develop future prediction criteria.

Differences in the reference standards (eg, in the samples, stimuli, assay used and cut-offs used) highlighted problems with our reference standard for T1D. However, although cut-offs were derived in a variety of ways, they were largely comparable and appropriate for detecting insulin deficiency in the populations of interest. Where more than one cut-off was used, ${ }^{24262730}$ this made little difference $(<12 \%)$ to the proportion of patients classified and the cut-offs identified. These differences represent potential issues with using our 'gold standard' for insulin deficiency when aiming to classify T2D. We would therefore suggest caution in future studies when classifying patients close to the proposed C-peptide cut-off.

\section{Other forms of diabetes}

We have only considered the two main forms of diabetes for which there are clear national and international treatment guidelines. Rarer subtypes are not considered here. Other forms of diabetes, such as latent autoimmune diabetes of adults, are not included in international guidelines and appropriate treatment would be guided by insulin deficiency, our gold standard. Further work would be needed to derive criteria for a 'grey area' where diagnosis of subtype is less certain and further investigations would be required to aid classification.

\section{Implications and future work}

Evidence-based guidelines on the classification of T1D and T2D need to include clinical criteria on how the diagnosis should be made. This is a major omission in current national and international guidelines for diabetes. The evidence as identified in this review suggests age at diagnosis and time to insulin (when available) are essential components as they contribute most to the predictive ability. BMI, and other clinical criteria, do not appear to add to add further discrimination. The criteria identified are similar to the RCGP/NHS Diabetes Guidelines for Classification ${ }^{13}$ which are based on consensus expert opinion. These guidelines would 
therefore, represent a suitable classification scheme until a stronger evidence base is available.

New studies are urgently needed to further develop and validate criteria suitable for classifying diabetes. We identified no studies in the Asian or paediatric populations, and only one study assessing features close to diagnosis. ${ }^{31}$ Determining classification rules for both the incident and prevalent population would be important. Labelling a patient's diabetes at the outset is crucial as the classification given is rarely reconsidered. The evidence in this review should be used to redevelop a clinical prediction tool for T1D and T2D. C-peptide is likely to be less discriminatory at diagnosis, as patients with T1D can still produce their own insulin in the 'honeymoon' period, so it would be important to examine predictors of insulin deficiency after this time. Future studies should be large-scale, prospective and give results for all racial and age groups using follow-up C-peptide measurements at least 3 years after diagnosis as an outcome. These studies would help answer if clinical criteria used in combination are sufficient to accurately classify diabetes, or whether investigations, such as islet autoantibodies, are needed in addition. Consideration of other forms of diabetes, such as monogenic diabetes, is also important.

We did not include antibodies in our search criteria as we limited our review to routinely available clinical criteria. Antibodies may represent a useful test at diagnosis, where C-peptide is of limited value due to the 'honeymoon period', where patients with T1D are still able to produce significant amounts of their own insulin for a short period of time. A systematic review examining the use of antibodies at predicting long-term insulin deficiency is presently in progress (Prospero reference CRD42012001736)

In conclusion, we have performed the first systematic review of the literature that examines using clinical criteria for the classification of diabetes. Although, only 11 studies were identified, age at diagnosis and time to insulin were consistent as discriminatory criteria across studies. BMI did not aid classification over these factors. The discriminatory criteria identified were similar to those proposed by the RCGP/NHS Diabetes Classification guidelines, so these would represent a suitable classification scheme at present. New studies are urgently needed to assess and validate the most appropriate clinical criteria. This review provides a summary of the current knowledge base for reference in any future studies developing classification rules.

Contributors BMS designed the review, carried out screening, reviewing, data extraction, quality assessment and synthesis, and led writing of the manuscript. She is guarantor. JL helped design the review, carried out screening, reviewing, data extraction, quality assessment and synthesis (independently, in parallel with BMS), and helped draft and revise the manuscript. CC designed the search strategy and performed the initial literature search and revised the draft manuscript. JL performed additional searching, retrieved full-text articles for review and revised the draft manuscript. BAK and AJ helped design the review, and revised the draft manuscript. RJP acted as third reviewer in cases of disagreement for the systematic review and revised the draft manuscript. CJH helped design the review, advised on synthesis and revised the draft manuscript. ATH helped design the review, advised on synthesis and revised the draft manuscript.

Funding This study was funded by the National Institute for Health Research (NIHR) under its Research for Patient Benefit (RfPB) programme (PB-PG-0711-25111) and supported by the National Institute for Health Research (NIHR) Collaboration for Leadership in Applied Health Research and Care South West Peninsula at the Royal Devon and Exeter NHS Foundation Trust. ATH and BMS are core members of the NIHR Exeter Clinical Research Facility. ATH is an NIHR Senior Investigator and a Wellcome Trust Senior Investigator. The views expressed are those of the author(s) and not necessarily those of the NHS, the NIHR or the Department of Health. The authors affirm their independence from these funders. The funders played no part in the study design, collection, analysis or interpretation of data or in the writing of the report, or in the decision to submit the article for publication.

Competing interests None declared.

Provenance and peer review Not commissioned; externally peer reviewed.

Data sharing statement No additional data are available.

Open Access This is an Open Access article distributed in accordance with the terms of the Creative Commons Attribution (CC BY 4.0) license, which permits others to distribute, remix, adapt and build upon this work, for commercial use, provided the original work is properly cited. See: http:// creativecommons.org/licenses/by/4.0/

\section{REFERENCES}

1. National Institute for Clinical Excellence. Clinical Guideline 15: type 1 diabetes: diagnosis and management of type 1 diabetes in children, young people and adults. London: NICE, 2010.

2. The National Collaborating Centre for Chronic Conditions. TYPE 2 DIABETES: national clinical guideline for management in primary and secondary care (update). London: NICE, 2011.

3. American Diabetes Association. Standards of medical care in diabetes-2013. Diabetes Care 2013;36(Suppl 1):S11-66.

4. Inzucchi SE, Bergenstal RM, Buse JB, et al. Management of hyperglycaemia in type 2 diabetes: a patient-centered approach. Position statement of the American Diabetes Association (ADA) and the European Association for the Study of Diabetes (EASD). Diabetologia 2012;55:1577-96.

5. DeWitt DE, Hirsch IB. Outpatient insulin therapy in type 1 and type 2 diabetes mellitus: scientific review. JAMA 2003;289:2254-64.

6. Yki-Jarvinen $\mathrm{H}$. Combination therapies with insulin in type 2 diabetes. Diabetes Care 2001;24:758-67.

7. Inzucchi SE, Bergenstal RM, Buse JB, et al. Management of hyperglycemia in type 2 diabetes: a patient-centered approach: position statement of the American Diabetes Association (ADA) and the European Association for the Study of Diabetes (EASD). Diabetes Care 2012;35:1364-79.

8. American Diabetes Association. Diagnosis and classification of diabetes mellitus. Diabetes Care 2010;33(Suppl 1):S62-9.

9. International Diabetes Federation. Definition and diagnosis of diabetes mellitus and intermediate hyperglycaemia: report of a WHO/IDF consultation. Geneva: World Health Organisation, 2006.

10. Jones AG, Hattersley AT. The clinical utility of C-peptide measurement in the care of patients with diabetes. Diabet Med 2013;30:803-17.

11. Sacks DB, Arnold M, Bakris GL, et al. Guidelines and recommendations for laboratory analysis in the diagnosis and management of diabetes mellitus. Clin Chem 2011;57:e1-47.

12. Farmer A, Fox R. Diagnosis, classification, and treatment of diabetes. BMJ 2011;342:d3319.

13. Royal College of General Practitioners and NHS Diabetes. Coding, Classification and Diagnosis of Diabetes. Secondary Coding, Classification and Diagnosis of Diabetes. 2011. http://www.sdrn.org. uk/sites/sdrn.org.uk/files/nhs\%20diagnosis\%20classification\% 20report.pdf

14. de Lusignan S, Khunti K, Belsey J, et al. A method of identifying and correcting miscoding, misclassification and misdiagnosis in diabetes: a pilot and validation study of routinely collected data. Diabet Med 2010;27:203-9.

15. Seidu S, Davies MJ, Mostafa S, et al. Prevalence and characteristics in coding, classification and diagnosis of diabetes in primary care. Postgrad Med J 2014;90:13-17. 
16. Sabbah E, Savola K, Ebeling T, et al. Genetic, autoimmune, and clinical characteristics of childhood- and adult-onset type 1 diabetes. Diabetes Care 2000;23:1326-32.

17. Turner R, Stratton I, Horton V, et al. UKPDS 25: autoantibodies to islet-cell cytoplasm and glutamic acid decarboxylase for prediction of insulin requirement in type 2 diabetes. UK Prospective Diabetes Study Group. Lancet 1997;350:1288-93.

18. Shields BM, Peters JL, Cooper $\mathrm{C}$, et al. Identifying clinical criteria to predict Type 1 diabetes, as defined by absolute insulin deficiency: a systematic review protocol. BMJ Open 2012;2:pii: e002309.

19. No authors listed]. Classification and diagnosis of diabetes mellitus and other categories of glucose intolerance. National Diabetes Data Group. Diabetes 1979;28:1039-57.

20. Whiting PF, Rutjes AW, Westwood ME, et al. QUADAS-2: a revised tool for the quality assessment of diagnostic accuracy studies. Ann Intern Med 2011;155:529-36.

21. Balasubramanyam A, Garza G, Rodriguez L, et al. Accuracy and predictive value of classification schemes for ketosis-prone diabetes. Diabetes Care 2006;29:2575-9.

22. Benhamou PY, Marwah T, Balducci F, et al. Classification of diabetes in patients with end-stage renal disease. Validation of clinical criteria according to fasting plasma C-peptide. Clin Nephrol 1992;38:239-44.

23. Welborn TA, Garcia-Webb $\mathrm{P}$, Bonser A, et al. Clinical criteria that reflect $C$-peptide status in idiopathic diabetes. Diabetes Care 1983:6:315-16.

24. Laakso M, Sarlund H, Pyorala K. Clinical characteristics in the discrimination between patients with low or high $\mathrm{C}$-peptide level among middle-aged insulin-treated diabetics. Diabetes Res 1987;4:95-9.

25. Boyle JP, Engelgau MM, Thompson TJ, et al. Estimating prevalence of type 1 and type 2 diabetes in a population of African Americans with diabetes mellitus. Am J Epidemiol 1999;149:55-63.

26. Service FJ, Rizza RA, Zimmerman BR, et al. The classification of diabetes by clinical and C-peptide criteria. A prospective population-based study. Diabetes Care 1997;20:198-201.

27. Prior MJ, Prout T, Miller D, et al. C-peptide and the classification of diabetes mellitus patients in the Early Treatment Diabetic Retinopathy Study. Report number 6. The ETDRS Research Group. Ann Epidemiol 1993;3:9-17.

28. Welborn TA, Webb PG, Bonser AM. Basal C-peptide in the discrimination of type I from type II diabetes. Diabetes Care 1981;4:616-19.

29. Shields BM, Shepherd MH, Buphati-Raju N, et al. Clinical criteria do not precisely classify which insulin treated patients have type 1 diabetes. Diabetic Med 2010;27(Supp S1):29.

30. Nielsen NV, Tronier B. C-peptide in diabetes mellitus treated with insulin. A 3-year epidemiological study on the island of Falster Denmark. Diabetes Res 1986;3:475-8.

31. Ekpebegh C, Longo-Mbenza B, Blanco-Blanco E. Islet immunity and beta cell reserve of indigenous Black South Africans with ketoacidosis at initial diagnosis of diabetes. Ethn Dis 2013;23:196-201.

32. Whiting PF, Rutjes AW, Westwood ME, et al. A systematic review classifies sources of bias and variation in diagnostic test accuracy studies. J Clin Epidemiol 2013;66:1093-104. 